\title{
Trace Selection for Improved WLAN Monitoring
}

\author{
Matteo Sammarco \\ UPMC Sorbonne Universités \\ Paris, France \\ matteo.sammarco@lip6.fr
}

\author{
Miguel Elias M. Campista \\ Universidade Federal do Rio \\ de Janeiro \\ Rio de Janeiro, Brazil \\ miguel@gta.ufrj.br
}

\author{
Marcelo Dias de Amorim \\ UPMC Sorbonne Universités \\ Paris, France \\ marcelo.amorim@lip6.fr
}

\begin{abstract}
Existing measurement techniques for IEEE 802.11-based networks assume that the higher the density of monitors in the target area, the higher the quality of the measure. This assumption is, however, too strict if we consider the cost involved in monitor installation and the necessary time to collect and merge all traces. In this paper, we investigate the balance between number of traces and completeness of collected data. We propose a method based on similarity to rank collected traces according to their contribution to the monitoring system. With this method, we are able to select only a subset of traces and still keep the quality of the measure, while improving system scalability. In addition, based on the same rank, we identify monitors that can be relocated to enlarge the monitored area and increase the overall efficiency of the system. Finally, our experimental results show that the proposed solution leads to a better tradeoff in terms of unique captured frames over the number of merge operations.
\end{abstract}

\section{Categories and Subject Descriptors}

C.2.3 [Computer-communication Networks]: Network Operations - network monitoring, network management

\section{General Terms}

Management, Design, Measurement, Experimentation

\section{Keywords}

Wireless networks; IEEE 802.11; measurement; monitoring; scalability

\section{INTRODUCTION}

Wireless monitoring systems typically employ multiple passive sensing nodes scattered out across an area of interest to capture as much information as possible. The need for multiple monitors covering the same area comes from the fact that

Permission to make digital or hard copies of all or part of this work for personal or classroom use is granted without fee provided that copies are not made or distributed for profit or commercial advantage and that copies bear this notice and the full citation on the first page. Copyrights for components of this work owned by others than ACM must be honored. Abstracting with credit is permitted. To copy otherwise, or republish, to post on servers or to redistribute to lists, requires prior specific permission and/or a fee. Request permissions from permissions@acm.org.

HotPlanet'13, August 16, 2013, Hong Kong, China.

Copyright 2013 ACM 978-1-4503-2177-8/13/08 ...\$15.00. packet sniffers may miss events as a consequence of physical issues, such as fading, interference, collisions, and hardware outages. The outcome of such a process is a collection of traces, each one collected by a different monitor, which are merged into a single file. The merged trace provides a better picture of the wireless activity in the target area at the cost of increased overhead to capture more traces. This procedure brings up then a tradeoff between completeness and scalability $[3,12]$.

Much effort has been devoted to large scale monitoring systems and merging techniques. Initially, traces were collected from logs generated in access points or captured in adjacent wired networks $[1,7]$. These solutions provide a narrowed view of the area of interest because they failed to monitor the entire wireless network. Cheng et al. claim that the dynamics of a wireless environment can be only rebuilt if all frames and delivery outcomes are captured [3]. Alternative approaches rely on as many distributed passive sniffers as possible. These systems use a central entity in charge of generating merged traces taking into account synchronization issues [3, 9]. A common characteristic of all these solutions is that they are concerned with the contrasting requirements of completeness of the captured traces and scalability of the monitoring systems [8, 13, 14]. Although these systems are sophisticated, their main goal is simply to merge as many traces as possible, rather than improving the system efficiency by previously selecting the most relevant traces.

In this paper, we investigate the actual need of completely merging all the obtained traces. To accomplish that, we rank traces according to their individual contributions, which are computed considering pairwise trace similarity. In fact, different traces are likely to bring their own specific observations; when such observations are not significant (i.e., traces have a high level of similarity), some traces may not be considered in the merging procedure. In our experiments, we observe a clear tradeoff between the amount of information obtained with an additional trace and system scalability. In a nutshell, our contributions can be summarized as follows:

- Similarity analysis. We propose two metrics to analyze the similarity between IEEE 802.11 traces, called intra- and inter-flow similarity. The first one is based on the ratio of frames captured by both traces and the total number of frames. The latter, on the other hand, is based on the ratio of flows observed in both traces and the total number of flows.

- Trace ranking. We propose a ranking method, called "Hamiltonian", to sort individual traces according to 
their contribution to the merged trace. Based on the similarity metrics, we can select a subset of traces to be merged and also we can decide whether another trace is needed to correctly monitor a given area of interest.

- Scalability gains. We show results attesting that our method leads to significant scalability improvements, meaning that we can detect whether the contribution of a given monitor is irrelevant with regard to its location. If so, it can be moved further, enlarging the monitored area.

This paper is structured as follows. In Section 2, we present our experimental scenarios as well as the hardware and software used. In Section 3, we introduce the proposed intra- and inter-flow metrics and we show their impact. In Section 4, we propose the method to smarter choose a subset of traces and we present the improvements obtained on the merging procedure. Our conclusions and future works are reported in Section 5.

\section{SNIFFING AND EXPERIMENTAL SETUP}

We adopt WiPal as the network sniffing tool $[4,5]$. It is, actually, both a software library and a set of tools to provide flexible trace manipulation. Apart from the capture feature, WiPal can also identify reference frames, perform trace synchronization, merge, concatenation, and extract sub-traces or single fields. It also has a module for statistic presentation. Given two traces $t_{i}$ and $t_{j}$, WiPal operates in three steps:

- Identifying reference frames. Beacon frames and non-retransmitted probe response are considered as unique frames by WiPal. They embed 64-bit timestamps (not related to the next synchronization step) and are extracted from the input traces and then intersected. The intersection process first puts every unique frame of $t_{i}$ in a hash table, then it does the same for unique frames in $t_{j}$; if a collision occurs, a reference frame is found.

- Synchronization. Synchronizing two traces means mapping timestamps of one trace to values that are compatible with results obtained from the second trace. WiPal operates on windows of $w+1$ reference frames and, for each of them $\left(R_{i}\right)$, the process performs linear regression using reference frames in the relative window $R_{i-\lfloor w / 2\rfloor}, \ldots, R_{i+\lceil w / 2\rceil}$. Once the reference frames are synchronized, the two traces are also synchronized accordingly.

- Merging. Frames from synchronized traces are copied to the output trace avoiding duplicates.

We have conducted experiments in two scenarios. We call the first one IRCICA, as we deployed our monitors at the second floor of the IRCICA/LIFL computer science laboratory of Lille; and the second one INRIA as we conducted our experiments at the INRIA National Institute for Research in Computer Science and Control building also in Lille. Figure 1(a) shows the placement of monitors along the corridor at IRCICA (leading to a linear shape). Note that monitors 1 to 6 are equally spaced, while monitors 7 and 8 are slightly separated from the others. At INRIA, as shown in

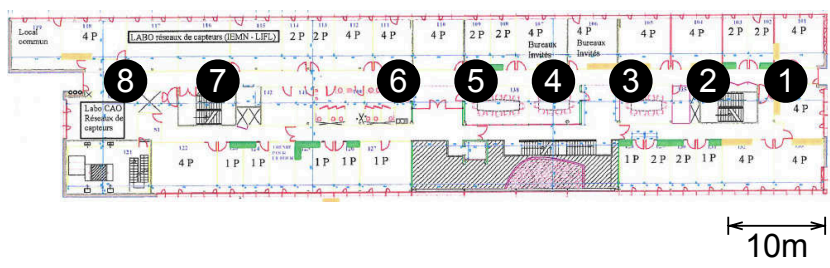

(a) IRCICA scenario.

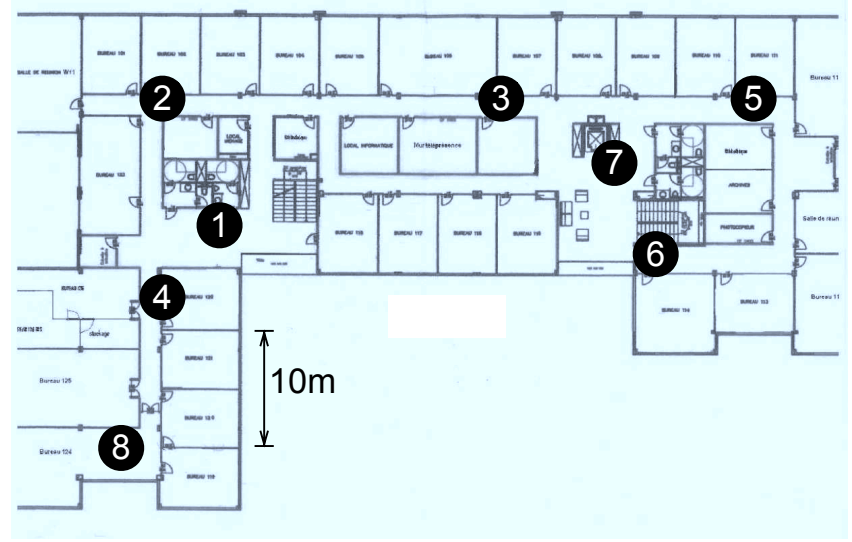

(b) INRIA scenario.

Figure 1: Monitor deployment for both scenarios.

Figure 1(b), monitors are placed along the L-shaped floor. Monitors cannot directly view each other, as they are separated by walls.

In both scenarios, the monitors sniffed the wireless network activity during 100 minutes, collecting IEEE 802.11b/g frames. Each monitor produced only one trace, listening to channel 1. The traces have an average size of 205 and 110 MByte at IRCICA and INRIA scenarios, respectively. In our experiments, each frame is limited to 220 bytes and the MAC addresses are anonymous.

Frame losses always occur independent from the sniffer hardware and software configuration [11]. In our experiments, we use Asus EEEPC-4G netbooks as sniffers. They are equipped with 512-MByte RAM and three USB Wi-Fi Netgear WG111v3 cards as wireless network adapters. The operating system is a Xandros OS with a customized kernel.

\section{TRACE SIMILARITY}

Hereinafter, we denote $\mathcal{T}=\left\{t_{0}, \ldots, t_{n}\right\}$ the set of traces captured in the same time period, where $t_{i}$ is the trace collected by monitor $s_{i}$. We consider that each trace is composed of flows of frames. We denote by $f_{i}^{m}$ the $m^{\text {th }}$ source-destination flow in trace $t_{i}$ and $p_{i}^{n}$ the $n^{t h}$ frame in $t_{i}$. We denote the cardinality of a set by $|\bullet|$.

We propose two metrics to discover how similar two traces are. The intra-flow similarity computes the ratio of the number of frames simultaneously captured by two given monitors nodes over the total number of frames captured by them. The inter-flow similarity, on the other hand, considers the intersection of flows instead of frames. Therefore, it is proportional to the ratio between the number of flows "observed" by the two monitors over the total number of flows 
"observed" by them. A flow is considered "observed" if at least one of its frames is captured. In addition, since flows can have different numbers of frames, we give them different weights; the larger the number of frames in a flow, the more important it is.

\subsection{Intra-flow similarity}

We use the Jaccard similarity index to compute the intraflow similarity. This metric considers all the frames captured by two sniffers, digging into each source-destination flow. This is why we consider this metric as intra-flow. Considering two captured traces $t_{i}$ and $t_{j}$ as a set of frames, $t_{i}=\left\{p_{i}^{0}, \ldots, p_{i}^{n}\right\}$, the Jaccard similarity is:

$$
\mathrm{J}\left(t_{i}, t_{j}\right)=\frac{\left|t_{i} \cap t_{j}\right|}{\left|t_{i} \cup t_{j}\right|}
$$

Figure 2(a) depicts the intra-flow similarity matrix of the traces from IRCICA. Each point $(i, j)$ is gradually colored according to its value of $\mathrm{J}\left(t_{i}, t_{j}\right)$. As the monitors have been sequentially placed, higher values are on the diagonal. In the figure, we can identify three geographical regions: a central region (monitors 4-5-6) and two side regions (monitors 1-2-3 and 7-8). As monitors 7-8 are slightly isolated from the others (see Figure 1(a)), they present a high similarity between them and low similarity with all the others. This means that small changes in monitors' geographical placement have a big impact on the amount of original data captured. In the INRIA scenario, shown in Figure 3(a), traces 1 and 2 share a low intra-flow similarity with all the others and the remaining traces are split into two sets, 3-5-6-7 and 4-8.

\subsection{Inter-flow similarity}

From a higher point of view, it is worth considering the flows in common between two traces. The importance of each flow is proportional to the number of frames it has. Let us consider $\mathcal{T}$ the corpus of traces and each unique flow $f_{i}^{m}$ as a single term in a trace $t_{i}$. We weight the importance of the flow using the Term Frequency-Inverse Document Frequency (TF-IDF) metric [10]. TF-IDF is widely used in the information retrieval and text mining field to weight the similarity between two text documents. In our context, documents are traces and terms are flows. The inter-flow similarity between two traces $t_{i}$ and $t_{j}$ is computed as follows. If the union of the two traces has $l$ distinct flows, a TF-IDF vector of $l$ elements is assigned to each trace. Each element of the vector is the product of two factors. The first one is the flow frequency in that trace. The second one is the logarithm of the inverse trace (the one containing that flow) frequency over all the traces in the corpus.

$$
\operatorname{TF}-\operatorname{IDF}\left(t_{i}, l\right)=\frac{\left|f_{i \cup j}^{l}\right|}{\left|t_{i}\right|} \cdot \log \frac{|\mathcal{T}|}{\left|\left\{t_{i} \in \mathcal{T} \mid f_{i \cup j}^{l} \in t_{i}\right\}\right|} .
$$

The inter-flow similarity (IFS) is a value in the range $[0 ; 1]$ (from orthogonal traces to equal traces), given by the cosine of the angle between these vectors. This is equal to the dot product of the vectors, divided by the product of their magnitude:

$$
\operatorname{IFS}\left(t_{i}, t_{j}\right)=\frac{\sum_{l} \operatorname{TF}-\operatorname{IDF}\left(t_{i}, l\right) \cdot \operatorname{TF}-\operatorname{IDF}\left(t_{j}, l\right)}{\sqrt{\sum_{l} \mathrm{TF}-\mathrm{IDF}\left(t_{i}, l\right)^{2}} \cdot \sqrt{\sum_{l} \mathrm{TF}-\mathrm{IDF}\left(t_{j}, l\right)^{2}}} .
$$

In Figures 2(b) and 3(b), we can observe how the interflow similarity metric can clarify the relationship between traces. In the IRCICA scenario, Figure 2(b), we can clearly distinguish a first cluster of high similarity traces from monitors 7-8, which correspond to the monitors located on the west side of the building. Because they are slightly separated from the other monitors, they present low similarity. Central monitors 4-5-6 compose another cluster, while on the east side of the building, we have a set of monitors 2-3 and a singleton with monitor 1 . This last monitor produces a trace with a perceptible similarity with trace 2 fading down up to trace 6 . We remark that, even if very geographically close, trace pairs 1-2 and 3-4 do not present a very high similarity among them.

Figure 3(b) shows that the traces from monitors 3-5-6-7, placed in the east side of the INRIA building (Figure 1(b)), have a very high inter-flow similarity as well as traces from monitors 2-4. Monitors 1 and 8 constitute two singletons.

\section{MERGING EFFICIENCY}

Deploying a large-scale WLAN monitoring system raises two important issues. First, deployment cost can be significant depending on the number of monitors. Second, it has a direct consequence on the amount of data to be postprocessed. Moving such a bulk of data is an additional shortcoming, since it impacts on the underlying network. There is then a tradeoff to respect between representativeness and scale.

Merging traces from different monitors is based on the assumption that a monitor might capture an event that another monitor misses. Merging together many traces, however, is a CPU- and time-consuming process. The procedure is recursive and uses pairs of traces as input - with $n$ traces, it would require $n-1$ steps to synchronize, merge, and rewrite the final trace. Moreover, depending on the sequence of traces to merge, this procedure converges faster or slower as similar traces (i.e., traces with several equal frames) do not contribute with unique frames when merged together. To tackle this issue, we propose a method to better chose the sequence of traces to merge. At the end, we show that the procedure improves system scalability without losing monitoring information.

\subsection{Trace selection strategy}

We consider the matrix of inter-frame similarity (IFS) values calculated in Section 3.2 and shown in Figures 2(b) and $3(\mathrm{~b})$ as an adjacent matrix of a fully connected graph $G(V, E)$. In this graph, each vertex $v_{i}$ corresponds to a captured trace $t_{i}$ and each edge $e_{i j}$ has a weight linearly proportional to the IFS between traces $t_{i}$ and $t_{j}$ connected $\left(\operatorname{IFS}\left(t_{i}, t_{j}\right)\right)$. For the sake of visualization, we consider the length of each edge as proportional to its weight. We then use the Force Atlas algorithm embedded in the Gephi graph visualization and manipulation software to plot the graph [2]. Figures 2(c) and 2(c) show how nodes are arranged on a plane.

We observe that pairs of vertices with high similarity are likely placed farther away, whereas vertices with lower similarity are placed closer. Our hypothesis is that, touching all the nodes according to the minimum Hamiltonian path, is a smarter way to iteratively select traces to merge because it ranks the traces according to their contribution to the final merge. This rank is obtained from the path sequence, which 


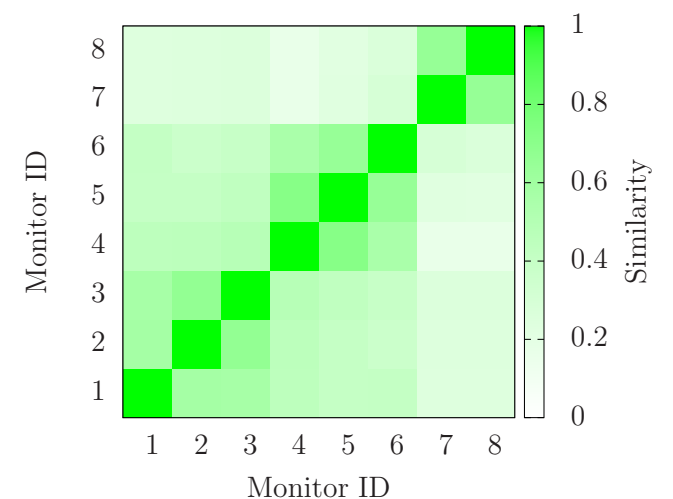

(a) Intra-flow similarity matrix.

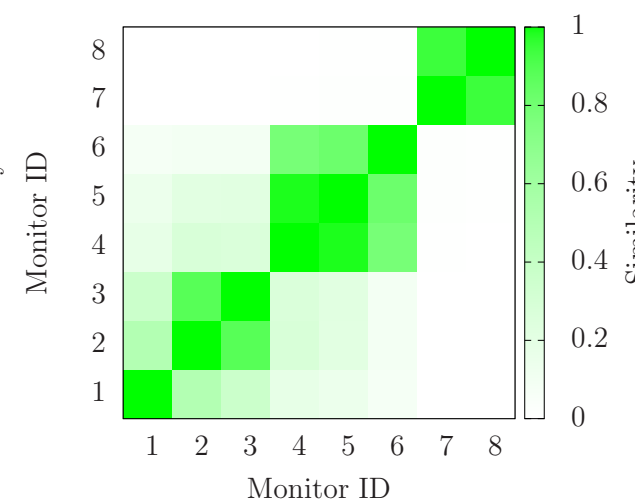

(b) Inter-flow similarity matrix.

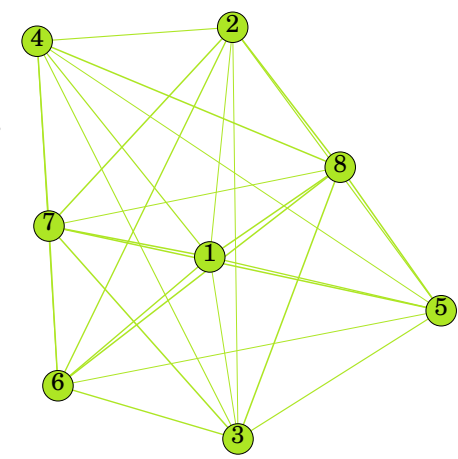

(c) Inter-flow similarity graph.

Figure 2: IRCICA scenario. (a) and (b) are, respectively, the intra- and inter-flow similarity matrices between traces, whereas (c) is the graph generated using traces as nodes and inter-flow similarity values as edge lengths.



(a) Intra-flow similarity matrix.

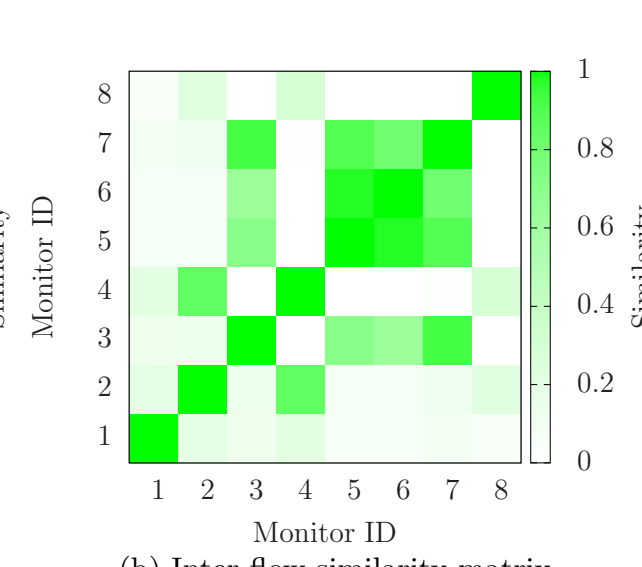

(b) Inter-flow similarity matrix.

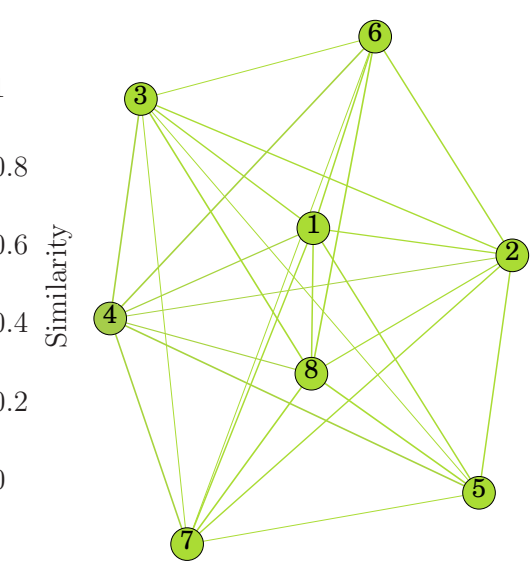

(c) Inter-flow similarity graph.

Figure 3: INRIA scenario. (a) and (b) are, respectively, the intra- and inter-flow similarity matrices between traces, whereas (c) is the graph generated using traces as nodes and inter-flow similarity values as edge lengths.

is the solution of the Hamiltonian path problem. Traces at the beginning of the path contribute more to the merge in terms of number of unique frames, whereas traces at the end give a not relevant contribution. The full connectivity guarantees the existence of such a Hamiltonian path. We refer to Hamiltonian path or Hamiltonian sequence equivalently.

At the end of the procedure, we can select a subset of traces with higher contribution to be merged and we can also identify the monitors not satisfactorily contributing to the system. Merging a subset of traces can improve the system scalability while moving nodes to other points where they can have a higher contribution can enlarge the monitored area. We calculate the optimal Hamiltonian path with Concorde TSP Solver [6].

\subsection{Evaluation}

We evaluate our ranking strategy comparing its performance with a sequential strategy. As a sequential strategy, we mean merging the traces starting from monitor 1 and monitor 2 until monitor 8 . We use this sequence because, as far as we know, there is no other work proposing a dif- ferent method in the literature. Hence, we assume that the sequence is randomly chosen. For comparison purposes, we find the minimum Hamiltonian path starting from the trace captured by monitor 1 in both scenarios. It is worth mentioning that the utilization of a different method was considered such as using the trace size or the amount of frames per trace, but the differences among them are not appreciable.

For the IRCICA scenario we get the Hamiltonian path 1-5-8-2-4-7-6-3. In Figure 4(a), we can appreciate how fast this sequence converges to the total covering of unique captured frames. We show the percentage of unique frames added to the final trace per merge procedure. As the first merge, the Hamiltonian sequence chooses trace 5 instead of trace 2, resulting in a positive percentage difference of $25 \%$ compared with the sequential strategy. At the second merge, the difference between trace 8 and 3, raises to $32 \%$. From the third merge operation on the gap starts reducing. The Hamiltonian curve remains always on the top of the sequential one, proving the importance of the right trace selection at the beginning of the whole merging process. Indeed, the first three chosen traces, 1-5-8, geographically cover all the 

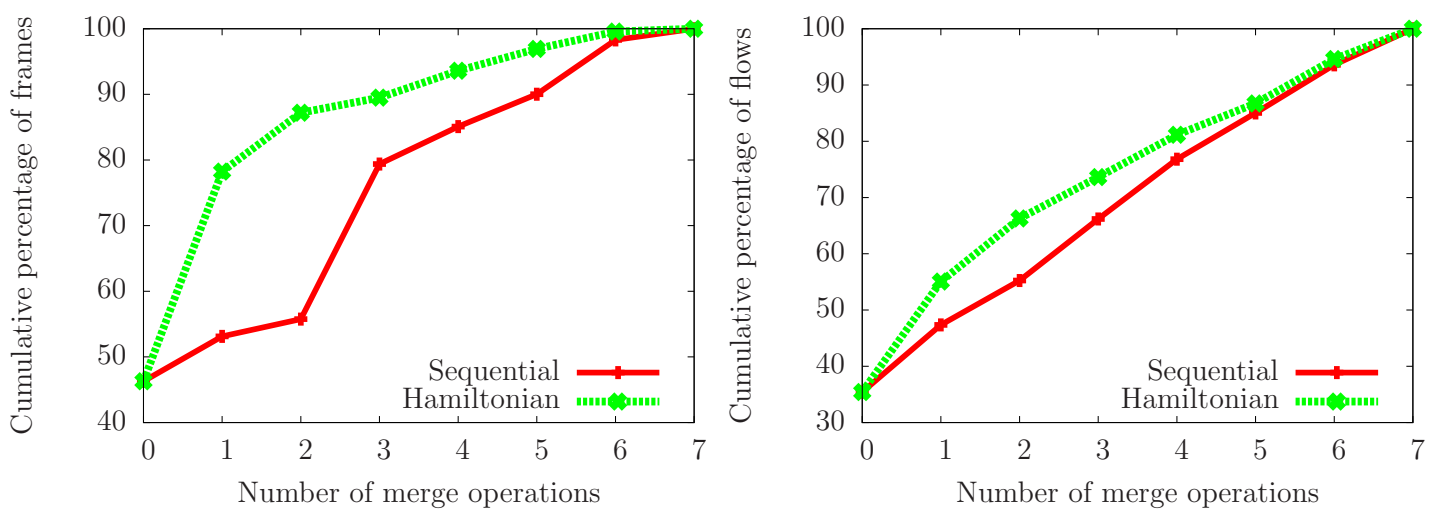

(a) New unique frames found over number of merge (b) New unique flows found over number of merge operations. operations.

Figure 4: IRCICA scenario. Comparison between the Hamiltonian merging sequence (1-5-8-2-4-7-6-3) and the sequential merging order.
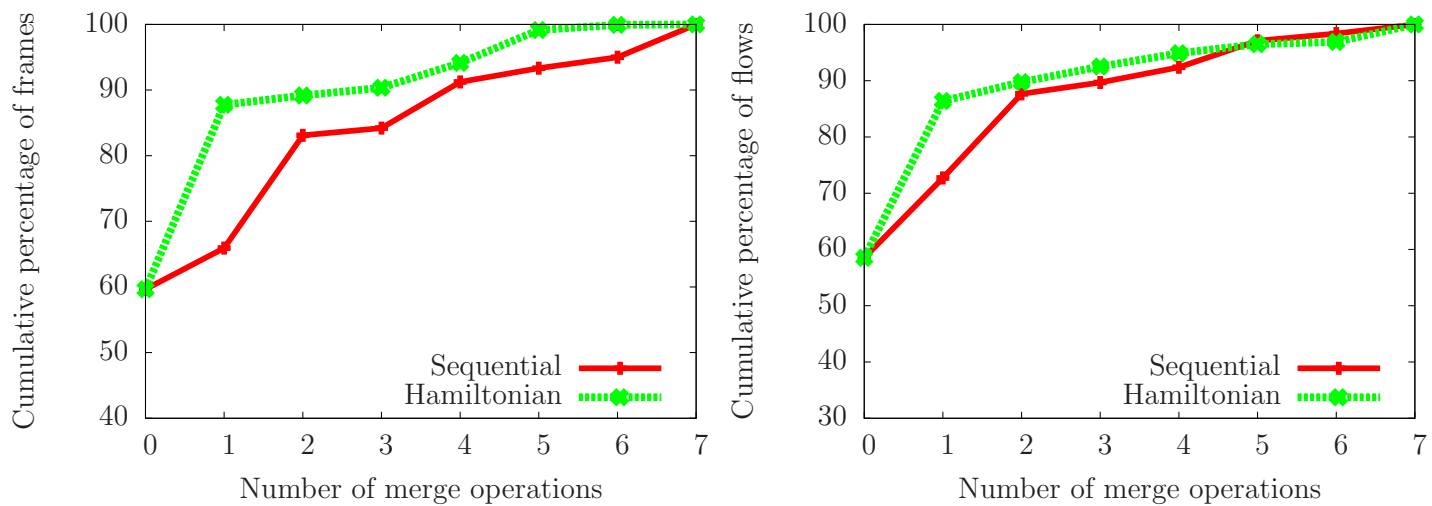

(a) New unique frames found over number of merge (b) New unique flows found over number of merge operations.

operations.

Figure 5: INRIA scenario. Comparison between the Hamiltonian merging sequence (1-6-3-4-7-8-5-2) and the sequential merging order.

area and $87 \%$ of all the captured frames. In addition, if the traces merged at steps 3-4-5-6 can still add a small amount of new frames, the last merged trace (trace 3) does not generate a significant increment. This suggests that its utilization is not relevant and could be left aside or it suggests that monitor 3 should be better moved somewhere else.

Regarding the quantity of unique flows detected, the Hamiltonian selection strategy presents a faster covering. Figure 4(b) shows the percentage of unique flows added to the final trace per merge procedure. The largest differences compared with the sequential strategy are at the firsts merging steps: $9 \%$ higher at the first step and $11 \%$ higher at the second. Comparing Figures 4(a) and 4(b), we also remark that flows chosen at the beginning are the most significant ones contributing with a large number of frames. From the third merging step on, $27 \%$ of new flows are detected with only $12 \%$ of new unique frames.

The INRIA scenario also shows that the Hamiltonian strategy leads to a better performance compared with the sequential one, even when monitors are not geographically placed respecting the sequential order. In this case, the trace sequence given by the Hamiltonian path is 1-6-3-4-7-8-5-2.
Figure 5(a) shows the performance of the Hamiltonian strategy in INRIA scenario. Again, the largest difference appears at the first steps. Choosing to merge traces 1 and 6 , instead of 1 and 2, we have an increment of $22 \%$ in the percentage of unique frames captured. We also note that, choosing the Hamiltonian sequence, the last two monitors, do not produce a significant improvement. These monitors could be better moved somewhere else to enlarge the monitored area without a significant loss.

Figure 5(b) shows that even considering steps 5 and 6 , where the sequential procedure detects more flows, the Hamiltonian strategy still detects more unique frames. The sequential strategy detects more flows in these steps because most frames are merged at the very beginning of the whole procedure.

\section{SUMMARY AND OUTLOOK}

In this work we show how to better scale current monitoring systems, selecting the monitors which produce the most significant contribution for the merging procedure. Starting from a set of captured traces, we first compute the interflow similarity between all of them. Then, we model the 
network as a graph to find the most appropriate sequence of merges. This sequence is found using the minimum Hamiltonian path, computed on such graph, which sorts the traces according to their contribution to the final merged file. Our approach leads to two main improvements. The system can become more scalable, since the merges can be limited to a subset of traces at the beginning of the sequence; and the system can become larger, since last traces in the sequence can suggest moving the monitors to other areas.

Although the inter-flow similarity looks like to have a good impact on the trace selection, we wish to exploit other similarity metrics. We used the optimum Hamiltonian path starting from trace 1 in order to compare it with a sequential merging. A further improvement could be the utilization of the absolute minimum path.

\section{Acknowledgments}

Matteo Sammarco and Marcelo Dias de Amorim carried out part of the work at LINCS (http://www.lincs.fr). Miguel Campista would like to thank CAPES, CNPq, FAPERJ, and FINEP for their support. This work was partially funded by the French National Research Agency (ANR) under the project ANR VERSO RESCUE (ANR-10-VERS-003).

\section{REFERENCES}

[1] A. Balachandran, G. M. Voelker, P. Bahl, and P. V. Rangan. Characterizing user behavior and network performance in a public wireless LAN. In $A C M$ SIGMETRICS, pages 195-205, June15-19 2002.

[2] M. Bastian, S. Heymann, and M. Jacomy. Gephi: an open source software for exploring and manipulating networks. 2009. In International AAAI Conference on Weblogs and Social Media, 2011.

[3] Y.-C. Cheng, J. Bellardo, P. Benkö, A. C. Snoeren, G. M. Voelker, and S. Savage. Jigsaw: solving the puzzle of enterprise 802.11 analysis. In $A C M$ SIGCOMM, pages 39-50, Sept.11-15 2006.

[4] T. Claveirole and M. D. de Amorim. WiPal: IEEE 802.11 traces manipulation software, Jan. 2010.
[5] T. Claveirole and M. D. de Amorim. Manipulating Wi-Fi packet traces with WiPal: design and experience. Software Practice \& Experience, 42(5):585-599, May 2012.

[6] W. Cook. Concorde TSP solver. See: http://www. tsp. gatech. edu/concorde. html, 2005.

[7] T. Henderson, D. Kotz, and I. Abyzov. The changing usage of a mature campus-wide wireless network. In ACM MobiCom, pages 187-201, Sep.-Oct.26-1 2004.

[8] C. C. Ho, K. N. Ramachandran, K. C. Almeroth, and E. M. Belding-Royer. A scalable framework for wireless network monitoring. In Proceedings of the 2nd ACM international workshop on Wireless mobile applications and services on WLAN hotspots, WMASH '04, pages 93-101, New York, NY, USA, 2004. ACM.

[9] R. Mahajan, M. Rodrig, D. Wetherall, and J. Zahorjan. Analyzing the MAC-level behavior of wireless networks in the wild. In ACM SIGCOMM, pages 75-86, Sept.11-15 2006.

[10] J. Ramos. Using TF-IDF to determine word relevance in document queries. In Proceedings of the First Instructional Conference on Machine Learning, 2003.

[11] P. Serrano, M. Zink, and J. Kurose. Assessing the Fidelity of COTS 802.11 Sniffers. In INFOCOM 2009, IEEE, pages 1089-1097, April.

[12] Y. Sheng, G. Chen, H. Yin, K. Tan, U. Deshpande, B. Vance, D. Kotz, A. Campbell, C. McDonald, T. Henderson, and J. Wright. MAP: a scalable monitoring system for dependable 802.11 wireless networks. Wireless Communications, IEEE, 15(5):10-18, October.

[13] K. Tan, C. McDonald, B. Vance, C. Arackaparambil, S. Bratus, and D. Kotz. From MAP to DIST: the evolution of a large-scale WLAN monitoring system. IEEE Transactions on Mobile Computing, 99(PrePrints):1, 2012.

[14] J. Yeo, M. Youssef, and A. Agrawala. A framework for wireless lan monitoring and its applications, 2004. 